(CPAP) and noninvasive intermittent positivepressure ventilation (NIPPV) - in patients with acute cardiogenic pulmonary edema.

The 3CPO trial involved patients with a clinical diagnosis of acute cardiogenic pulmonary edema who were admitted to 26 hospital emergency departments in the UK. Patients had a respiration rate $>20$ breaths per min, arterial hydrogen-ion concentration $>45 \mathrm{nmol} / \mathrm{l}$, and pulmonary edema on chest radiography. Patients were elderly (mean age 77.7 years) and the cohort had a high prevalence of ischemic heart disease, hypertension, tachycardia, hypercapnia, and acidosis. Patients were randomly allocated to receive standard oxygen therapy $(n=367)$, CPAP $(n=346)$, or NIPPV $(n=356)$ in addition to concurrent medical therapy. No significant difference in 7-day mortality was evident between the standard oxygen therapy and noninvasive ventilation groups (9.5\% vs $9.8 \%)$. Similar rates of intubation or death within 7 days were observed in the NIPPV and CPAP groups $(11.1 \%$ vs $11.7 \%)$. Noninvasive ventilation was, however, associated with significant improvements in pulse rate, dyspnea, acidosis, and hypercapnia compared with standard ventilation.

The investigators recommended that CPAP or NIPPV be used in patients with drugrefractory cardiogenic pulmonary edema who experience severe respiratory distress.

Original article Gray A et al. (2008) Noninvasive ventilation in acute cardiogenic pulmonary edema. N Engl J Med 359: $142-151$

\section{Robotic catheter ablation safely eliminates atrial arrhythmia in humans}

The success of manual catheter ablation of arrhythmogenic foci depends on the operator's skill and experience. Saliba and colleagues' preliminary study in humans has demonstrated that remote-controlled robotic catheter ablation of atrial fibrillation (AF) is feasible and safe. This approach could overcome the limitations of manual catheter manipulation.

This prospective study enrolled 40 ablationnaive patients (mean \pm SD age $57 \pm 20$ years, 29 men) with drug-refractory AF, from three European medical centers. First, the hollow catheter sheath of the Sensei Robotic Catheter
System ${ }^{\circledR}$ (Hansen Medical, Mountain View, CA) was manually positioned in the inferior right atrium. The sheath was then robotically steered into the left atrium via a transeptal puncture and the ablation catheter inserted. Ablation of the pulmonary vein antra continued under robotic control until the pulmonary veins and superior vena cava were electrically isolated. Two patients developed pericardial tamponade during pulmonary-vein isolation, which resolved with pericardiocentesis. The 23 patients who had atrial flutter as well as AF additionally underwent ablation of the posterior cavotricuspid isthmus. After 1 year, 34 patients were free from atrial arrhythmias without the use of antiarrhythmic medication. No pulmonary vein stenoses were reported.

The authors concluded that the safety and efficacy of this robotic system is similar to that of conventional catheter ablation and recommend that a randomized, controlled trial of the two strategies be initiated.

Original article Saliba W et al. (2008) Atrial fibrillation ablation using a robotic catheter remote control system: initial human experience and long-term follow-up results. J Am Coll Cardiol 51: 2407-2411

\section{Lipoprotein(a) levels do not predict cardiovascular disease in women taking HRT}

Women with high levels of lipoprotein(a) have an increased risk of cardiovascular disease, but few medications or lifestyle changes are proven to decrease lipoprotein(a) levels. To date, studies of the effects of hormone replacement therapy (HRT) on lipoprotein(a) levels have shown conflicting results; most studies that show an HRT-associated decrease in lipoprotein(a) levels have been underpowered to assess any effects on cardiovascular disease.

Danik and colleagues have studied 27,736 women ( $\geq 45$ years of age) to determine whether HRT affects the relationship between lipoprotein(a) levels and cardiovascular disease. At the start of the study all these women were considered healthy; however, those taking HRT had lower lipoprotein(a) and LDL levels, and higher total cholesterol, HDL, triglyceride and C-reactive protein levels than women not receiving HRT. Furthermore, women 